\title{
No association between triple-negative breast cancer and prognosis of patients receiving breast-conserving treatment
}

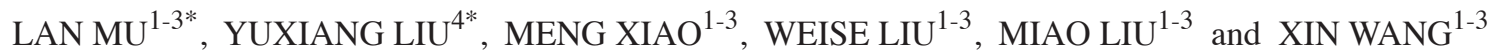 \\ ${ }^{1}$ First Department of Breast Surgery, Tianjin Medical University Cancer Institute and Hospital, \\ National Clinical Research Center for Cancer, Tianjin's Clinical Research Center for Cancer; ${ }^{2}$ Key \\ Laboratory of Cancer Prevention and Therapy, Tianjin's Clinical Research Center for Cancer; \\ ${ }^{3}$ Key Laboratory of Breast Cancer Prevention and Therapy, Ministry of Education, Tianjin 300060; \\ ${ }^{4} 2011$ Collaborative Innovation Center of Tianjin for Medical Epigenetics, Key Laboratory of Hormones and \\ Development (Ministry of Health), Metabolic Diseases Hospital, Tianjin Medical University, Tianjin 300070, P.R. China
}

Received August 18,2015; Accepted April 13, 2017

DOI: $10.3892 / 01.2017 .7251$

\begin{abstract}
The role of triple-negative breast cancer (TNBC) in breast-conserving treatment is controversial. The present study aimed at evaluating the prognosis of patients with TNBC following breast-conserving treatment (BCT) within 5 years. The present study investigated a cohort of 757 patients with early stage breast cancer, diagnosed and treated with BCT between January 2002 and March 2010 at Tianjin Medical University Cancer Institute and Hospital. The patients were divided into three groups according to receptor expression: Estrogen receptor (ER) or progesterone receptor (PR)-positive; epidermal grow th factor receptor 2 (HER2)-enriched: ER and PR negative but HER2-positive; TNBC: ER, PR and HER2 receptor-negative. The primary endpoint was recurrence or mortality within 5 years after breast cancer diagnosis. Multivariable Cox analysis was used to determine the risk of locoregional relapse, distant
\end{abstract}

Correspondence to: Dr Xin Wang, First Department of Breast Surgery, Tianjin Medical University Cancer Institute and Hospital, National Clinical Research Center for Cancer, Tianjin's Clinical Research Center for Cancer, Binshui Road, Tianjin 300060, P.R. China

E-mail: wangxintjzlyy@yeah.net

*Contributed equally

Abbreviations: TNBC, triple-negative breast cancer; ER, estrogen receptor; PR, progesterone receptor; HER2, human epidermal growth factor receptor 2; BCT, breast-conserving treatment; LRR, locoregional recurrence; LRFS, locoregional recurrence-free survival; DMFS, distant metastasis-free survival; DFS, disease-free survival; OS, overall survival; HR, hazard ratio; CI, confidence interval

Key words: triple-negative breast cancer, breast-conserving treatment, recurrence, metastasis, mortality metastases, total relapse and mortality associated with the intrinsic subtypes. Of the 757 patients with status of all receptors available, 541 (71.5\%) were luminal, 66 (8.7\%) were HER2-enriched and 150 (19.8\%) were TNBC. Patients with TNBC were more likely to have histological grade III tumors $(27.3 \%)$ compared with luminal $(8.3 \%)$ and HER2-enriched $(16.7 \%)$ subtypes $(\mathrm{P}<0.001)$. Within 5 years, locoregional recurrence rate was 2.4, 7.6 and $7.3 \%$ for luminal, HER2-enriched and TNBC, respectively $(\mathrm{P}=0.005)$. Mortality rate was $2.2,9.1$ and $4.7 \%$ for luminal, HER2-enriched and TNBC, respectively $(\mathrm{P}=0.007)$. There was no significant difference in rates of distant metastases $(\mathrm{P}=0.164)$ and total relapse $(\mathrm{P}=0.138)$. TNBC was not an independent prognostic predictor for women treated with BCT within 5 years after breast cancer diagnosis on multivariate analysis. Patients with TNBC were not at significantly increased 5-year risks of locoregional recurrence, distant metastasis, total relapse or mortality at so remain appropriate candidates for BCT.

\section{Introduction}

Breast cancer is a heterogeneous disease and divided into different subtypes according to estrogen receptor (ER), progesterone receptor (PR), and human epidermal growth factor receptor 2 (HER2) status $(1,2)$. Triple-negative breast cancer (TNBC), which is characterized by the lack of ER, PR and HER2 expression, has attracted substantial attention $(3,4)$. There is a significant overlap between TNBC and basal-like breast cancer. Therefore, TNBC and basal-like breast cancer are frequently regarded as synonymous $(5,6)$. TNBC is assessed by immunohistochemistry, and basal-like breast cancer is identified using gene expression profiling (7). TNBCs are fast-growing tumors and tend to metastasize compared with other types of breast cancer (8). TNBC has consistently been associated with poor clinical outcomes in radical mastectomy due to its invasive characteristics, and poor response to hormone therapy and HER2-targeted therapy (9-11).

Similar survival rates of women following breast-conserving treatment (BCT) and radical mastectomy have been 
demonstrated $(12,13)$. Consequently, BCT has become a preferable option for early stage breast cancer patients (14). However, due to the aggressive characteristics of TNBC, whether patients with TNBC are suitable for BCT remains controversial. A number of studies have reported that following BCT women with TNBC had a higher rate of local failure compared with women with other breast cancer subtypes $(15,16)$. Investigators reported that TNBC was associated with an increased risk of distant metastasis, but it was not associated with increased locoregional recurrence (LRR) (17). However, Millar et al (18) reported that there was no increase in distant metastasis risk following $\mathrm{BCT}$ in patients with TNBC compared with patients with other breast cancer phenotypes. Additionally, Gangi et al (19) demonstrated that patients with TNBC were not associated with increased local relapse compared with patients with non-TNBC subtypes following BCT; however, they found that the TNBC phenotype was associated with shorter overall survival (OS).

Taken together, the role of TNBC in BCT remains unclear. The outcomes of patients with TNBC following BCT have not been well described. Therefore, an investigation was conducted to retrospectively compare the 5-year outcomes of women with TNBC to women with other subtypes breast cancer following $\mathrm{BCT}$ and to evaluate the prognostic value of TNBC in patients with BCT.

\section{Patients and methods}

Patients. Between January 2002 and March 2010, a total of 757 patients with early-stage breast cancer treated with BCT at Tianjin Medical University Cancer Institute and Hospital were identified to meet the inclusion criteria. The inclusion criteria were as follows: i) Full details of ER, PR and HER2 status and ii) patients had with stage I/II/III breast cancer and treated by lumpectomy. Exclusion criteria included: i) males with breast cancer; ii) T4 disease; iii) stage IV disease; iv) previously underwent mastectomy and v) unknown ER, PR or HER2 status. Data collected included standard prognostic factors, such as age, menopausal status, tumor size, lymph node, ER, PR, and HER2 status, histological grade, date of surgery, adjuvant treatment received, time of LRR and metastatic progression, date of last follow-up (March 2015) and mortality. In the present study, the age of breast cancer patients ranged between 19 and 83 years, and the median age was 45 years. The pathological samples were taken as part of routine examination and biopsy, and the specimens were obtained on the day of operation performed, the number of specimens obtained varied between 6 and 20, according to different circumstances. The patients were staged using the sixth edition of the American Joint Committee on Cancer (AJCC) staging manual (20). The Elston-Ellis modification of Scarff-Bloom-Richardson (SBR) grading system was used for histological grade of breast cancer (21). Negative pathological margins were defined as no invasive carcinoma or ductal carcinoma in situ (DCIS) present at the margins; positive margins were defined as the presence of invasive carcinoma or DCIS at the margin. Ethical approval was obtained from the Tianjin Medical University Hospital (grant no. bc2017008), and written informed consent was obtained from all patients.
Formalin-fixed paraffin-embedded tissues. Samples obtained from each patient were formalin-fixed and paraffin-embedded as part of routine examination. In the study, a 4-mm-thick tissue specimen was cut from breast tumor for formalin fixation processes. Each sample was placed into standard tissue cassettes and completely submerged in a container filled with $4 \%$ neutral buffered formaldehyde for $24 \mathrm{~h}$ at room temperature. The formaldehyde-fixed samples were embedded in paraffin after stepwise dehydration in 70, 80, 90 and $99 \%$ ethanol, followed by isopropanol and xylene.

Immunohistochemistry (IHC) and fluorescent in situ hybridization (FISH). IHC and FISH analysis were conducted on tissue sections (3-4 $\mu \mathrm{m})$ and tissue microarray slides. IHC (Benchmark XT; Ventana Medical Systems, Inc., Tucson, AZ, USA) was performed on formalin-fixed and paraffin-embedded tissues of 757 patients using the avidin-biotin-immunoperoxidase technique (22). Tissue sections were deparaffinized in xylene and rehydrated in a graded series of ethanol. Antigen-retrieval was performed in citrate buffer $(\mathrm{pH} 6.0)$ at $120^{\circ} \mathrm{C}$ for $2.5 \mathrm{~min}$. Subsequently, the slides were exposed to $3 \%$ hydrogen peroxide for $20 \mathrm{~min}$ and washed with PBS for 5 min 3 times. The further blocking of tissues was performed with normal goat serum (Abcam Inc., Cambridge, $\mathrm{UK}$ ) for $30 \mathrm{~min}$ at room temperature. The sections were then incubated overnight at $4^{\circ} \mathrm{C}$ with the following primary antibodies: ER (cat no. NCL-L-PGR-312; dilution, 1:100), PR (cat no. NCL-L-ER-6F11; dilution, 1:80) (both Novocastra; Leica Biosystems GmbH, Wetzlar, Germany), HER2 (cat no. 800-2996; dilution 1:300; Ventana Medical Systems, Inc.). Following 5 washes with PBS, the slides were incubated with biotin-conjugated secondary antibody (cat no. ZB-2010; dilution, 1:200; OriGene Technologies, Inc., Beijing, China) for $30 \mathrm{~min}$ at room temperature. The sections with positive expression level was used as the positive control, the negative control was established with the primary antibody replaced by PBS. Detection was done by utilizing iView DAB Detection kit (Ventana Medical Systems, Inc.).

FISH analysis was conducted in line with the protocol of Abbott/Vysis PathVysion HER2 DNA Probe kit (cat no. 30161060/02J01-030; Abbott Molecular, Inc., Des Plaines, IL, USA), the Spectrum Orange fluorophore-labeled DNA probe and Spectrum Green fluorophore-labeled $\alpha$-satellite DNA probes from this kit were used to assess the HER2 gene locus and chromosome 17, respectively. In total, 2 separate fields of $\geq 20$ cells were counted and the average of the results of the selected tumor areas were used to calculate mean gene and chromosome 17 counts, which were used to calculate the ratio of HER2:CEP17 signal. Tumor cells from matching sites of IHC were scored for the number of red (HER2) and green (chromosome 17) signals. The slides were evaluated using an Olympus BX51 microscope (Olympus Corporation, Tokyo, Japan) with an oil-immersion objective lens and an appropriate filter set at a magnification of x100. Immunoreactivity was assessed independently by $\geq 2$ pathologists.

Subtype definitions. The patients were divided into three subtypes according to receptor expression: i) Luminal: ER or PR-positive, ii) HER2-enriched: ER and PR-negative but HER2 receptor-positive and iii) TNBC:ER, PR and 
HER 2-negative. ER, PR and HER2 data were obtained through routine clinical testing. The samples were defined as ER or PR-positive if $>1 \%$ of the cells were positive for immunohistostaining. HER2 immunoreactivity was assessed using a standardized score from $0-3$, based on the intensity of staining of the cell membrane and the proportion of tumor cells stained. The no staining or $<10 \%$ of tumor cells was estimated as negative (score, 0 ), the weak and incomplete staining of the membrane in $>10 \%$ of tumor cells was considered negative (score, $1+$ ), the weak to moderate complete staining of the membrane in $>10 \%$ tumor cells was evaluated as $2+$, and strong complete staining of the membrane in $>10 \%$ of tumor cells was determined to be positive (score, $3+$ ). Samples with 2+ were needed to be detected by FISH test, and the HER2 gene was considered to be amplified when the ratio of HER2:CEP17 in tumor cells was $>2.0$. Patients with negative ER and PR status and HER2 immunohistostaining score of $2+$ but no fluorescence in situ hybridization results were excluded in the present analysis.

Treatment delivery. All patients in the present study were treated surgically with breast-conserving surgery. A total of 675 patients had completed breast radiation therapy. Breast plus supraclavicular fossa radiation therapy was performed on 61 patients. A total of 21 patients did not undergo radiation treatment. Adjuvant systemic chemotherapy and hormone therapy were performed according to standard practices during that time interval. The majority of patients in the present study received anthracycline/taxane-based or cyclophosphamide, methotrexate and 5-fluorouracil chemotherapy regimens, and a minority of patients chose other chemotherapy regimens. It was recommended that patients with ER- and/or PR-positive disease who were premenopausal received tamoxifen treatment; postmenopausal patients chose aromatase inhibitors. Of the 757 patients with breast cancer, 717 received adjuvant systemic chemotherapy and 493 received hormone therapy. In total, 3 patients in the present study received trastuzumab therapy (data not shown).

Follow-up and outcomes. Follow-up has been maintained by reviewing clinical charts and by contacting patients by telephone or mail. Factors analyzed included clinical (age, menopause status, tumor-node-metastasis stage, tumor size), pathological (lymph node status, histological grade, pathological subtype and final margin status) and treatment (systemic therapy). The end-point of the present study was 5-year LRR, distant metastasis or mortality, and the study was ended when breast cancer mortality occurred. Total recurrence referred to LRR or distant metastasis. LRR-free survival (LRFS) was defined as the time from diagnosis to development of LRR (recurrence within the breast and regional relapse, including ipsilateral supraclavicular fossa, axilla or internal mammary lymph nodes). Distant metastasis-free survival (DMFS) was defined as the time from pathological diagnosis to the time from first evidence of distant metastasis. Disease-free survival (DFS) defined as the time of diagnosis to development of first evidence of metastasis or LRR. OS was defined as from the time of diagnosis to last follow-up or time of mortality from breast cancer (patients who succumbed to other causes were considered censored from the time of mortality).

Statistical analysis. All statistical analyses were carried out using SPSS 17.0 (SPSS, Inc., Chicago, IL, USA). The Kaplan-Meier method was used for univariate analysis and calculating LRFS, DMFS, DFS and OS, and the significance was assessed using the log-rank test. Cox proportional hazards regression analysis was used for multivariate analysis. Factors with a significance of $\mathrm{P}<0.10$ in univariate analysis were included in the multivariate Cox model. Differences between categorical variables were calculated using the $\chi^{2}$ test. $\mathrm{P}<0.05$ was considered to indicate a statistically significant difference.

\section{Results}

Patients. Of the 757 patients diagnosed with early breast cancer and treated with BCT in whom the status of all three markers (ER, PR, and HER2) were available, 541 (71.5\%) were sorted as luminal subtype, $66(8.7 \%)$ were defined as HER2-enriched subtype and 150 (19.8\%) were classified as TNBC subtype (Table I). The follow-up time ranged from 13 to 157 months, and the median follow-up time was 83 months. The 5-year LRFS, DMFS, DFS and OS for the whole cohort were $96.2,94.3,92.9$ and $96.7 \%$, respectively.

Clinicopathological characteristics according to different subtypes. Patient characteristics and results of statistical comparisons according to different breast cancer subtypes were summarized in Table I. The distribution of young patients ( $\leq 35$ years) was significantly different between luminal (13.1\%), HER2-enriched (27.3\%) and TNBC (19.3\%) subtypes, $(\mathrm{P}=0.016$; Table I). Patients with TNBC were more likely to have histological grade III tumors $(27.3 \%)$ compared with the luminal (8.3\%) and HER2 (16.7\%) subtypes $(\mathrm{P}<0.001$; Table I). Selection of chemotherapy treatment was also different among the 3 groups $(\mathrm{P}=0.021$; Table I).

5-year outcomes of breast cancer patients following BCT according to different subtypes. The 5-year outcomes of the three different breast cancer subtypes following BCT were different. The rate of 5-year LRR was significantly different between luminal (2.4\%), HER2-enriched (7.6\%) and TNBC $(7.3 \%)$ groups $(\mathrm{P}=0.005$; Table II). The rate of 5-year mortality was 2.2, 9.1 and 4.7\% for luminal, HER2-enriched and TNBC, respectively $(\mathrm{P}=0.007$; Table $\mathrm{II})$. There was no significant difference in the rates of distant metastases $(\mathrm{P}=0.164)$ and total relapse ( $\mathrm{P}=0.138$; Table II). The 5-year LRFS was 97.6, 92.4 and $92.7 \%$ for luminal, HER2-enriched and TNBC groups, respectively ( $\mathrm{P}=0.005$; Fig. 1A). The 5-year DMFS was 95.0, 89.4 and $94.0 \%$ for luminal, HER2-enriched and TNBC groups, respectively $(\mathrm{P}=0.164$; Fig. $1 \mathrm{~B})$. The 5 -year DFS was 93.9, 87.9 and 91.3\% for luminal, HER2-enriched and TNBC groups, respectively $(\mathrm{P}=0.138$; Fig. $1 \mathrm{C})$. The 5-year OS was 97.8, 90.9 and $95.3 \%$ for luminal, HER2-enriched and TNBC groups, respectively ( $\mathrm{P}=0.007$; Fig. 1D).

Univariate analysis of the prognostic factors associated with 5 -year outcomes of patients following BCT. The prognostic factors associated with 5-year LRFS, DMFS, DFS and OS in 
Table I. Distribution of clinical and treatment characteristics among different types patients with breast-conserving treatment.

\begin{tabular}{|c|c|c|c|c|}
\hline Parameters & Luminal, n (\%) & HER2-enriched, n (\%) & TNBC, n (\%) & P-value \\
\hline Total & $541(71.5)$ & $66(8.7)$ & $150(19.8)$ & \\
\hline Age at diagnosis, years & & & & 0.016 \\
\hline$\leq 35$ & $71(13.1)$ & $18(27.3)$ & $29(19.3)$ & \\
\hline $36-55$ & $368(68.0)$ & $39(59.1)$ & $100(66.7)$ & \\
\hline$>55$ & $102(18.9)$ & $9(13.6)$ & $21(14.0)$ & \\
\hline Menopausal status & & & & 0.676 \\
\hline Premenopausal & $369(68.2)$ & $48(72.7)$ & $100(66.7)$ & \\
\hline Postmenopausal & $172(31.8)$ & $18(27.3)$ & $50(33.3)$ & \\
\hline Number of positive LNs & & & & 0.937 \\
\hline 0 & $421(77.8)$ & $54(81.8)$ & $119(79.3)$ & \\
\hline $1-3$ & 97 (17.9) & $10(15.2)$ & $26(17.3)$ & \\
\hline$\geq 4$ & $23(4.3)$ & $2(3.0)$ & $5(3.3)$ & \\
\hline Tumor size, $\mathrm{cm}$ & & & & 0.316 \\
\hline $\mathrm{T} 1(\leq 2)$ & 378 (69.9) & $41(62.1)$ & $93(62.0)$ & \\
\hline $\mathrm{T} 2(>2, \leq 5)$ & $145(26.8)$ & $22(33.3)$ & $49(32.7)$ & \\
\hline $\mathrm{T} 3(>5)$ & $18(3.3)$ & $3(4.6)$ & $8(5.3)$ & \\
\hline TNM stage & & & & 0.601 \\
\hline I & $321(59.3)$ & $35(53.1)$ & $81(54.0)$ & \\
\hline II & $196(36.2)$ & $29(43.9)$ & $61(40.7)$ & \\
\hline III & $24(4.5)$ & $2(3.0)$ & $8(5.3)$ & \\
\hline Pathological subtype & & & & 0.566 \\
\hline IDC & $396(73.2)$ & $50(75.8)$ & $116(77.3)$ & \\
\hline Other & $145(26.8)$ & $16(24.2)$ & $34(22.7)$ & \\
\hline Histological grade & & & & $<0.001$ \\
\hline I & $106(19.6)$ & $5(7.6)$ & $16(10.7)$ & \\
\hline II & $344(63.6)$ & $41(62.1)$ & $81(54.0)$ & \\
\hline III & $45(8.3)$ & $11(16.7)$ & $41(27.3)$ & \\
\hline Unknown & $46(8.5)$ & $9(13.6)$ & $12(8.0)$ & \\
\hline Margin status & & & & 0.230 \\
\hline Positive & $12(2.2)$ & $0(0.0)$ & $1(0.7)$ & \\
\hline Negative & $529(97.8)$ & $66(100.0)$ & $149(99.3)$ & \\
\hline Chemotherapy & & & & 0.021 \\
\hline $\mathrm{CMF}$ & $89(16.4)$ & $21(31.8)$ & $23(15.3)$ & \\
\hline $\mathrm{A} / \mathrm{T}$ & 404 (74.7) & $39(59.1)$ & $119(79.4)$ & \\
\hline Other & $15(2.8)$ & $2(3.0)$ & $5(3.3)$ & \\
\hline No & $33(6.1)$ & $4(6.1)$ & $3(2.0)$ & \\
\hline Radiation & & & & 0.138 \\
\hline Breast & 477 (88.2) & $58(87.8)$ & $140(93.3)$ & \\
\hline Breast and SCF & $48(8.9)$ & $4(6.1)$ & $9(6.0)$ & \\
\hline No & $16(2.9)$ & $4(6.1)$ & $1(0.7)$ & \\
\hline Hormone therapy & & & & $<0.001$ \\
\hline Yes & $484(89.5)$ & $3(4.5)$ & $6(4.0)$ & \\
\hline No & $18(3.3)$ & $63(95.5)$ & $144(96.0)$ & \\
\hline Unknown & $39(7.2)$ & $0(0.0)$ & $0(0.0)$ & \\
\hline
\end{tabular}

HER2, human epidermal growth factor receptor 2; TNBC, triple-negative breast cancer; HER2, human epidermal growth factor receptor 2; LNs, lymph nodes; IDC, invasive ductal cancer; CMF, cyclophosphamide, methotrexate, 5-fluorouracil; A/T, anthracyclines/taxane; SCF, supraclavicular fossa; TNM, tumor-node-metastasis. 
Table II. 5-year outcomes of different subtype breast cancer patients with breast-conserving treatment.

\begin{tabular}{|c|c|c|c|c|}
\hline Parameters & Luminal, n (\%) & HER2 type, n (\%) & TNBC, n (\%) & P-value \\
\hline Total & $541(71.5)$ & $66(8.7)$ & $150(19.8)$ & \\
\hline LRR & & & & 0.005 \\
\hline Yes & $13(2.4)$ & $5(7.6)$ & $11(7.3)$ & \\
\hline No & $528(97.6)$ & $61(92.4)$ & $139(92.7)$ & \\
\hline Distant metastases & & & & 0.164 \\
\hline Yes & $27(5.0)$ & $7(10.6)$ & $9(6.0)$ & \\
\hline No & $514(95.0)$ & $59(89.4)$ & $141(94.0)$ & \\
\hline Total relapse & & & & 0.138 \\
\hline Yes & $33(6.1)$ & $8(12.1)$ & $13(8.7)$ & \\
\hline No & $508(93.9)$ & $58(87.9)$ & 137 (91.3) & \\
\hline Mortality & & & & 0.007 \\
\hline Yes & $12(2.2)$ & $6(9.1)$ & $7(4.7)$ & \\
\hline No & $529(97.8)$ & $60(90.9)$ & $143(95.3)$ & \\
\hline
\end{tabular}

HER2, human epidermal growth factor receptor 2; TNBC, triple-negative breast cancer; LRR, locoregional recurrence.

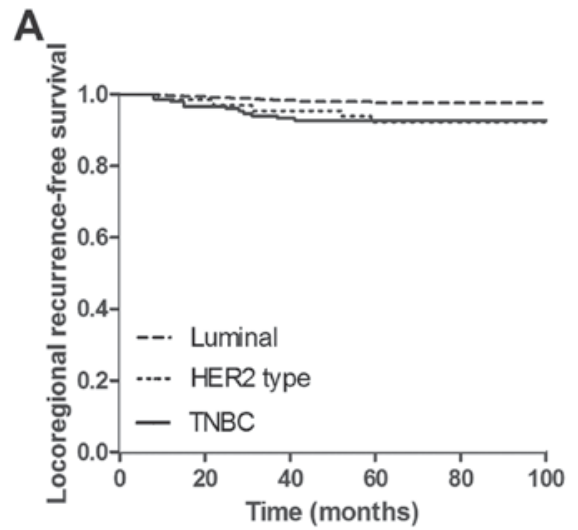

B
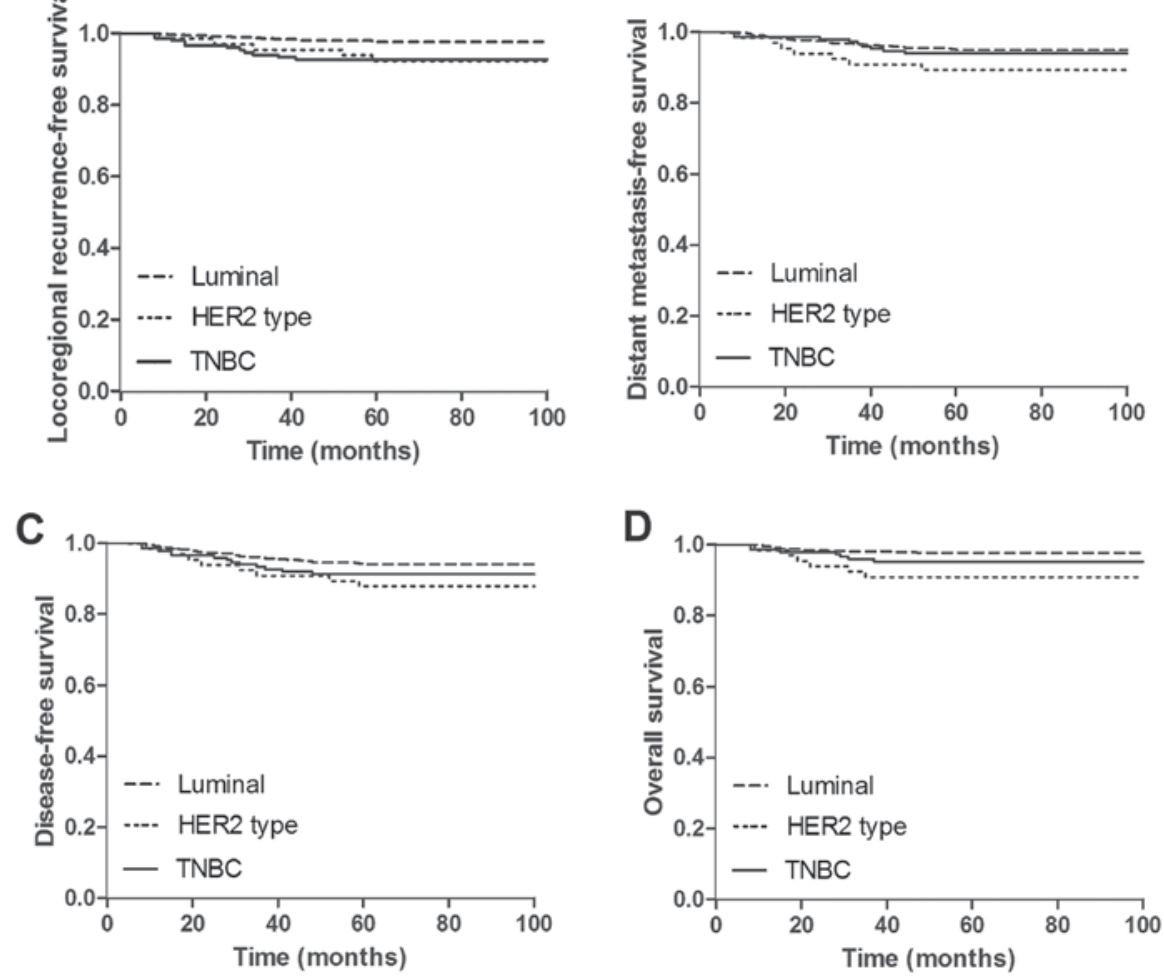

Figure 1. Kaplan-Meier analysis of 5-year survival outcomes of patients with BCT according to different breast cancer subtypes: (A) Locoregional recurrence-free survival; (B) distant metastasis-free survival; (C) disease-free survival and (D) overall survival probabilities. Statistically significant differences between the groups were determined using the log-rank test.

the whole cohort of patients following BCT were evaluated by univariate analysis. The results indicated that margin status $(\mathrm{P}=0.021)$, radiation therapy $(\mathrm{P}=0.031)$ and hormone therapy $(\mathrm{P}=0.008)$ may be associated with LRFS (Table III). Menopausal status $(\mathrm{P}=0.032)$, histological grade $(\mathrm{P}=0.049)$, margin status $(\mathrm{P}=0.004)$, chemotherapy treatment $(\mathrm{P}=0.001)$ and radiotherapy $(\mathrm{P}<0.001)$ may be prognostic factors for DMFS. Age at breast cancer diagnosis $(\mathrm{P}=0.029)$, histological grade $(\mathrm{P}=0.018)$, margin status $(\mathrm{P}<0.001)$, chemotherapy treatment $(\mathrm{P}=0.015)$, radiation therapy $(\mathrm{P}<0.001)$ and hormone therapy $(\mathrm{P}=0.046)$ may be associated with DFS. Factors associated with OS may be chemotherapy treatment $(\mathrm{P}=0.011)$, 
Table III. Univariate analysis of clinicopathological parameters for 5-year LRFS, DMFS, DFS and OS of patients with BCT.

\begin{tabular}{|c|c|c|c|c|c|c|c|c|c|}
\hline \multirow[b]{2}{*}{ Characteristics } & \multirow[b]{2}{*}{$\mathrm{n}$} & \multicolumn{2}{|c|}{ LRFS } & \multicolumn{2}{|c|}{ DMFS } & \multicolumn{2}{|c|}{ DFS } & \multicolumn{2}{|c|}{ OS } \\
\hline & & $\%$ & P-value & $\%$ & $\mathrm{P}$-value & $\%$ & P-value & $\%$ & P-value \\
\hline Age at diagnosis, years & & & 0.054 & & 0.142 & & 0.029 & & 0.256 \\
\hline$\leq 35$ & 118 & 92.4 & & 92.4 & & 88.1 & & 94.9 & \\
\hline $36-55$ & 507 & 97.0 & & 95.5 & & 94.5 & & 97.4 & \\
\hline$>55$ & 132 & 96.2 & & 91.7 & & 90.9 & & 95.5 & \\
\hline Menopausal status & & & 0.458 & & 0.032 & & 0.075 & & 0.362 \\
\hline Premenopausal & 517 & 96.5 & & 95.6 & & 94.0 & & 97.1 & \\
\hline Postmenopausal & 240 & 95.4 & & 91.7 & & 90.4 & & 95.8 & \\
\hline Number of positive LNs & & & 0.176 & & 0.169 & & 0.071 & & 0.177 \\
\hline 0 & 594 & 96.5 & & 95.1 & & 93.9 & & 97.3 & \\
\hline $1-3$ & 133 & 96.2 & & 91.0 & & 89.5 & & 94.7 & \\
\hline$\geq 4$ & 30 & 90.0 & & 93.3 & & 86.7 & & 93.3 & \\
\hline Tumor size, $\mathrm{cm}$ & & & 0.120 & & 0.191 & & 0.163 & & 0.225 \\
\hline $\mathrm{T} 1(\leq 2)$ & 512 & 96.1 & & 93.4 & & 92.4 & & 96.3 & \\
\hline $\mathrm{T} 2(>2, \leq 5)$ & 216 & 97.2 & & 96.8 & & 94.9 & & 98.1 & \\
\hline $\mathrm{T} 3(>5)$ & 29 & 89.7 & & 93.1 & & 86.2 & & 93.1 & \\
\hline TNM stage & & & 0.246 & & 0.995 & & 0.502 & & 0.667 \\
\hline I & 437 & 96.1 & & 94.3 & & 93.4 & & 96.8 & \\
\hline II & 286 & 96.9 & & 94.4 & & 92.7 & & 96.9 & \\
\hline III & 34 & 91.2 & & 94.1 & & 88.2 & & 94.1 & \\
\hline Pathological subtype & & & 0.814 & & 0.282 & & 0.553 & & 0.807 \\
\hline IDC & 562 & 96.3 & & 93.8 & & 92.5 & & 96.8 & \\
\hline Other & 195 & 95.9 & & 95.9 & & 93.8 & & 96.4 & \\
\hline Histological grade & & & 0.061 & & 0.049 & & 0.018 & & 0.193 \\
\hline $\mathrm{I}$ & 127 & 98.4 & & 98.4 & & 96.9 & & 99.2 & \\
\hline II & 466 & 96.4 & & 93.6 & & 92.7 & & 96.1 & \\
\hline III & 97 & 91.8 & & 90.7 & & 86.6 & & 94.8 & \\
\hline Unknown & 67 & 97.0 & & 97.0 & & 95.5 & & 98.5 & \\
\hline Margin & & & 0.021 & & 0.004 & & $<0.001$ & & 0.338 \\
\hline Positive & 13 & 84.6 & & 76.9 & & 61.5 & & 92.3 & \\
\hline Negative & 744 & 96.4 & & 94.6 & & 93.4 & & 96.8 & \\
\hline Chemotherapy & & & 0.558 & & 0.001 & & 0.015 & & 0.011 \\
\hline $\mathrm{CMF}$ & 133 & 95.5 & & 92.5 & & 91.0 & & 96.2 & \\
\hline $\mathrm{A} / \mathrm{T}$ & 562 & 96.6 & & 95.9 & & 94.3 & & 97.5 & \\
\hline Other & 22 & 95.5 & & 81.8 & & 85.0 & & 92.5 & \\
\hline None & 40 & 92.5 & & 85.0 & & 81.8 & & 86.4 & \\
\hline Radiation & & & 0.031 & & $<0.001$ & & $<0.001$ & & $<0.001$ \\
\hline Breast & 675 & 96.4 & & 95.3 & & 94.1 & & 97.2 & \\
\hline Breast and SCF & 61 & 96.7 & & 91.8 & & 88.5 & & 96.7 & \\
\hline No & 21 & 85.7 & & 71.4 & & 66.7 & & 81.0 & \\
\hline Hormone therapy & & & 0.008 & & 0.200 & & 0.046 & & 0.026 \\
\hline Yes & 493 & 97.6 & & 95.3 & & 94.3 & & 98.0 & \\
\hline No & 225 & 92.9 & & 92.0 & & 89.3 & & 94.2 & \\
\hline Unknown & 39 & 97.4 & & 94.9 & & 94.9 & & 94.9 & \\
\hline
\end{tabular}

LRFS, locoregional recurrence-free survival; DMFS, distant metastasis-free survival; DFS, disease-free survival; OS, overall survival; BCT, breast-conserving treatment; IDC, invasive ductal cancer; CMF, cyclophosphamide, methotrexate, 5-fluorouracil; A/T, anthracyclines/taxane; $\mathrm{SCF}$, supraclavicular fossa; TNM, tumor-node-metastasis. 
Table IV. Multivariate analysis of 5-year risks of LRR and distant metastases of patients with breast-conserving treatment.

\begin{tabular}{|c|c|c|c|c|c|c|}
\hline \multirow[b]{2}{*}{ Characteristics } & \multicolumn{3}{|c|}{ LRR } & \multicolumn{3}{|c|}{ Distant metastases } \\
\hline & HR & $95 \% \mathrm{CI}$ & P-value & HR & $95 \% \mathrm{CI}$ & P-value \\
\hline \multicolumn{7}{|l|}{ Molecular subtypes } \\
\hline TNBC & 1.000 & & & 1.000 & & \\
\hline Luminal & 0.316 & $0.043-2.317$ & 0.257 & 0.767 & $0.344-1.712$ & 0.517 \\
\hline HER2-enriched & 0.937 & $0.318-2.757$ & 0.906 & 1.492 & $0.540-4.126$ & 0.441 \\
\hline \multicolumn{7}{|c|}{ Age at diagnosis, years } \\
\hline$\leq 35$ & 1.000 & & & NA & & \\
\hline $36-55$ & 0.407 & 0.174-0.949 & 0.037 & & & \\
\hline$>55$ & 0.459 & $0.143-1.470$ & 0.190 & & & \\
\hline \multicolumn{7}{|l|}{ Menopausal status } \\
\hline Premenopausal & NA & & & 1.000 & & \\
\hline Postmenopausal & & & & 1.536 & $0.770-3.063$ & 0.223 \\
\hline \multicolumn{7}{|l|}{ Histological grade } \\
\hline I & 1.000 & & & 1.000 & & \\
\hline II & 2.345 & $0.534-10.291$ & 0.259 & 5.064 & $1.198-21.407$ & 0.027 \\
\hline III & 3.595 & $0.731-17.675$ & 0.115 & 6.408 & $1.351-30.397$ & 0.019 \\
\hline Unknown & 1.274 & $0.172-9.440$ & 0.813 & 1.487 & $0.202-10.939$ & 0.697 \\
\hline \multicolumn{7}{|l|}{ Margin } \\
\hline Positive & 1.000 & & & 1.000 & & \\
\hline Negative & 0.135 & $0.028-0.647$ & 0.012 & 0.210 & $0.059-0.746$ & 0.016 \\
\hline \multicolumn{7}{|l|}{ Chemotherapy } \\
\hline None & NA & & & 1.000 & & \\
\hline $\mathrm{CMF}$ & & & & 1.007 & $0.295-3.434$ & 0.992 \\
\hline $\mathrm{A} / \mathrm{T}$ & & & & 0.473 & $0.159-1.403$ & 0.177 \\
\hline Other & & & & 1.826 & $0.479-6.965$ & 0.378 \\
\hline \multicolumn{7}{|l|}{ Radiation therapy } \\
\hline None & 1.000 & & & 1.000 & & \\
\hline Breast & 0.178 & $0.050-0.638$ & 0.008 & 0.206 & $0.077-0.554$ & 0.002 \\
\hline Breast and SCF & 0.123 & $0.019-0.805$ & 0.029 & 0.338 & $0.090-1.266$ & 0.107 \\
\hline \multicolumn{7}{|l|}{ Hormone therapy } \\
\hline No & 1.000 & & & NA & & \\
\hline Yes & 1.081 & $0.152-7.697$ & 0.938 & & & \\
\hline Unknown & 1.119 & $0.069-18.065$ & 0.937 & & & \\
\hline
\end{tabular}

LRR, locoregional recurrence; HR, hazard ratio; CI, confidence interval; TNBC, triple-negative breast cancer; HER2, human epidermal growth factor receptor 2; NA, not applicable; CMF, cyclophosphamide, methotrexate, 5-fluorouracil; A/T, anthracyclines/taxane; SCF, supraclavicular fossa.

radiation therapy $(\mathrm{P}<0.001)$ and hormone therapy $(\mathrm{P}=0.026$; Table III).

Multivariate analysis of prognosis of TNBC for patients following BCT within 5 years. Multivariate Cox regression analysis included molecular subtype, age at diagnosis, menopausal status, lymph node status, histological grade, margin status, chemotherapy treatment, radiation and hormone therapy. Compared with patients with TNBC, the luminal subtype was not associated with a significant lower risks in LRR [hazard ratio (HR), 0.316; 95\% confidence interval (CI), 0.043-2.317; $\mathrm{P}=0.257$; Table IV)], distant metastasis (HR,
0.767; 95\% CI, 0.344-1.712; $\mathrm{P}=0.517$; Table IV), total relapse (HR, 1.623; 95\% CI, 0.475-5.539; $\mathrm{P}=0.440$; Table V) or breast cancer mortality (HR, 0.478; 95\% CI, 0.080-2.869; $\mathrm{P}=0.419$; Table V). HER2-enriched subtype was also not associated with reduced risks in LRR (HR, 0.937; 95\% CI, 0.318-2.757; $\mathrm{P}=0.906$; Table IV), distant metastasis (HR, 1.492; 95\% CI, 0.540-4.126; $\mathrm{P}=0.441$; Table IV), total relapse (HR, 1.277; 95\% CI, 0.512-3.186; $\mathrm{P}=0.600$; Table V) or breast cancer mortality (HR, 1.602; 95\% CI, 0.511-5.023; P=0.419; Table V) when contrasted to that of TNBC group. As a result, TNBC was not an independent prognostic predictor for patients with $\mathrm{BCT}$ in the present study. 
Table V. Multivariate analysis of 5-year risks of total relapse and breast cancer mortality of patients with breast-conserving treatment.

\begin{tabular}{|c|c|c|c|c|c|c|}
\hline \multirow[b]{2}{*}{ Characteristics } & \multicolumn{3}{|c|}{ Total relapse } & \multicolumn{3}{|c|}{ Breast cancer mortality } \\
\hline & HR & $95 \% \mathrm{CI}$ & P-value & HR & $95 \% \mathrm{CI}$ & P-value \\
\hline \multicolumn{7}{|l|}{ Molecular subtype } \\
\hline TNBC & 1.000 & & & 1.000 & & \\
\hline Luminal & 1.623 & $0.475-5.539$ & 0.440 & 0.478 & $0.080-2.869$ & 0.419 \\
\hline HER2-enriched & 1.277 & $0.512-3.186$ & 0.600 & 1.602 & $0.511-5.023$ & 0.419 \\
\hline \multicolumn{7}{|c|}{ Age at diagnosis, years } \\
\hline$\leq 35$ & 1.000 & & & NA & & \\
\hline $36-55$ & 0.359 & $0.173-0.747$ & 0.006 & & & \\
\hline$>55$ & 0.250 & $0.078-0.807$ & 0.020 & & & \\
\hline \multicolumn{7}{|l|}{ Menopausal status } \\
\hline Premenopausal & 1.000 & & & NA & & \\
\hline Postmenopausal & 2.133 & $0.972-4.682$ & 0.059 & & & \\
\hline \multicolumn{7}{|c|}{ Number of positive LNs } \\
\hline 0 & 1.000 & & & NA & & \\
\hline $1-3$ & 1.741 & $0.868-3.490$ & 0.118 & & & \\
\hline$\geq 4$ & 2.077 & $0.536-8.050$ & 0.290 & & & \\
\hline \multicolumn{7}{|l|}{ Histological grade } \\
\hline $\mathrm{I}$ & 1.000 & & & NA & & \\
\hline II & 2.532 & $0.885-7.247$ & 0.083 & & & \\
\hline III & 3.584 & $1.121-11.457$ & 0.031 & & & \\
\hline Unknown & 0.836 & $0.175-3.989$ & 0.823 & & & \\
\hline \multicolumn{7}{|l|}{ Margin status } \\
\hline Positive & 1.000 & & & NA & & \\
\hline Negative & 0.117 & $0.041-0.336$ & $<0.001$ & & & \\
\hline \multicolumn{7}{|l|}{ Chemotherapy } \\
\hline None & 1.000 & & & 1.000 & & \\
\hline $\mathrm{CMF}$ & 1.331 & $0.382-4.633$ & 0.653 & 0.611 & $0.134-2.784$ & 0.525 \\
\hline $\mathrm{A} / \mathrm{T}$ & 0.723 & $0.236-2.220$ & 0.571 & 0.496 & $0.126-1.960$ & 0.318 \\
\hline Other & 2.218 & $0.564-8.720$ & 0.254 & 1.877 & $0.355-9.921$ & 0.458 \\
\hline \multicolumn{7}{|l|}{ Radiation therapy } \\
\hline None & 1.000 & & & 1.000 & & \\
\hline Breast & 0.179 & $0.068-0.471$ & $<0.001$ & 0.219 & $0.066-0.729$ & 0.013 \\
\hline Breast and SCF & 0.174 & $0.041-0.740$ & 0.018 & 0.278 & $0.046-1.672$ & 0.162 \\
\hline \multicolumn{7}{|l|}{ Hormone therapy } \\
\hline No & 1.000 & & & 1.000 & & \\
\hline Yes & 0.455 & $0.147-1.407$ & 0.171 & 0.812 & $0.149-4.426$ & 0.810 \\
\hline Unknown & 0.326 & $0.056-1.902$ & 0.213 & 1.961 & $0.219-17.577$ & 0.547 \\
\hline
\end{tabular}

Total recurrence referred to locoregional recurrence or distant metastasis. TNBC, triple-negative breast cancer; HER2, human epidermal growth factor receptor 2; HR, hazard ratio; CI, confidence interval; NA, not applicable; CMF, cyclophosphamide, methotrexate, 5-fluorouracil; $\mathrm{A} / \mathrm{T}$, anthracyclines/taxane; SCF, supraclavicular fossa.

Other prognostic factors associated with 5-year outcomes for patients with BCT. In multivariate analysis, patients who were aged 36-55 years showed a reduced risk in 5-year LRR compared with patients $\leq 35$ years $(\mathrm{P}=0.037$; Table IV), and both patients aged 36-55 years and patients $>55$ years showed a inferior risk of total relapse compared with patients $\leq 35$ years
$(\mathrm{P}<0.05$; Tables IV and V). Patients with histological grade II or III tumors exhibited higher risk of distant metastasis $(\mathrm{P}<0.05$; Table IV), and patients with histological grade III tumors also showed an increased risk of total relapse $(\mathrm{P}=0.031$; Table $\mathrm{V})$ compared with patients with histological grade I tumors. Negative margin status was associated with reduced risks of 
LRR, distant metastasis and total relapse $(\mathrm{P}<0.05$; Tables IV and V) compared with positive margin status. Patients performed radiation therapy presented decreased risks in LRR, distant metastasis, total relapse and breast cancer mortality compared with patients without radiation therapy (Tables IV and $\mathrm{V})$.

\section{Discussion}

The associations between breast cancer types with different gene expression profiles and the prognosis of patients have recently been investigated (23). TNBC confers a poor clinical outcome to patients following BCT (24). Basal-like subtype has also been reported to be associated with an increased risk of local relapse and distant metastasis following BCT (15). Although basal-like subtype and TNBC are different breast cancer subtypes, it has been widely accepted that TNBC and basal-like breast tumors are synonymous (7).

In the current study, it was demonstrated that patients with TNBC presented different clinicopathological parameters compared with patients with luminal and HER2-enriched breast cancer. Patients with TNBC tended to have relatively high histological grades of tumor compared with luminal and HER2-enriched breast cancer patients. Consistent with this finding, previous studies have also reported that TNBC was more frequently associated with a higher histological grade $(8,24)$. Selection of chemotherapy treatment also differed between the 3 groups. The proportion of the patients that received chemotherapy was higher in the TNBC group than in other groups, which was consistent with the results of a previous study (25). Besides, patients with TNBC tended to choose anthracycline/taxane based chemotherapy regimens in the present study, although Kim et al (26) did not find similar results among luminal, HER2-enriched and TNBC groups.

However, there was no significant difference in prognosis between patients with TNBC and patients with luminal and HER2 subtypes following BCT. In the present study, the results of univariate analysis revealed that patients in the HER2-enriched subtype group exhibited reduced 5-year LRFS and OS, and rates of LRR and mortality were relatively high compared with the luminal and TNBC groups (i.e. the prognosis of patients with TNBC was not the poorest). Furthermore, results of multivariate analysis in our study indicated that TNBC was not associated with increased risks of 5-year LRR, distant metastasis, total relapse and mortality for patients with BCT. Similar to the present study, Gangi A et al (19) observed that TNBC was not associated with an increased rate of 5-year local relapse compared with non-TNBC subtypes in a retrospective study of 1851 patients with BCT. Additionally, Freedman et al (27) reported that the isolated 5-year LRR rate for patients with BCT was not different according to different breast cancer subtypes, and it was observed that there was a significantly higher rate of mortality in the HER2-enriched group compared with other subtypes. Findings in the present study indicated that HER2-enriched tumor was not an independent prognostic predictor for patients with BCT. The high rates of LRR and mortality in HER2-enriched subtype may be attributed to a small proportion of patients with positive HER 2 status that had received trastuzumab therapy. Another reason for these high rates might be that young patients ( $\leq 35$ years) who appeared to have a poorer prognosis (as confirmed in the present study) tended to be assigned to the HER2-enriched group and not the TNBC group. Noh et al (25) also demonstrated that despite the observation that TNBC and HER2-enriched subtypes were associated with younger age and higher histological grade, the rates of LRR and distant recurrence were not significantly different according to different molecular subtypes of breast cancer in patients following BCT.

To the best of our knowledge, the outcomes of TNBC patients with BCT remain contradictory. Solin et al (16) investigated 519 patients treated with BCT, and reported that patients with TNBC had an increased risk of local failure but a reduced risk of distant metastasis compared with other breast cancer subtypes. On the contrary, another investigation demonstrated that the TNBC subtype was associated with a higher risk of distant metastasis and mortality but was not associated with significantly higher rates of local relapse compared with other subtype (17). Kaplan et al (24) reported that patients with TNBC at a low risk of LRR had a significantly higher risk of distant metastasis compared with hormone receptor-positive/HER2-negative patients. Additionally, Braunstein et al (28) observed that TNBC was associated with reduced DFS following BCT in a retrospective study, which included 2,233 women who underwent BCT. In general, the issues regarding the role of TNBC in BCT remain unresolved. Encouragingly, the investigators have reported that TNBC patients with BCT had significantly lower LRR rate compared with patients treated with mastectomy (29). Results from a prospective randomized controlled multi-center trial indicate that for patients with TNBC following BCT, radiotherapy and chemotherapy exhibited significantly improved 5-year DFS and OS compared with those that received chemotherapy alone (30). Together with the findings from the previously mentioned reports and results from our study, patients with TNBC may remain appropriate candidates for BCT.

To date, publications evaluating the prognostic value of TNBC in BCT, demonstrated mixed results. There may be a number of reasons to account of these inconsistencies in findings. The term 'TNBC' has been used interchangeably with 'basal-like tumor'. The majority of TNBC are basal-like breast cancers, and the majority of basal-like tumors are also TNBC, which means that there is a considerable overlap between TNBC and basal-like tumors $(31,32)$. Additionally, TNBC has been subdivided into six distinct subtypes based on expression of various genes, including two basal-like subtypes, one with cell-cycle and DNA-damage-response gene expression signatures and the other enriched in growth factor signaling and myoepithelial markers; one immunomodulatory subtype; two mesenchymal subtypes with high expression of genes involved in differentiation and growth factor pathways; and one luminal androgen receptor subtype driven by androgen signaling $(4,33)$. For certain TNBC subtypes, mastectomy may lead to improved long-term outcomes compared with BCT (34). However, few studies differentiate basal-like subtype tumors from TNBC or clearly define the distinct TNBC subtypes $(32,35)$, which may result in contradictory outcomes following BCT reported in studies investigating patients with TNBC.

There are a number of limitations to the present study. Firstly, similar to other publications, owing to the limitations 
of detection techniques, examination instrumentation and cost, TNBC was not distinguished from basal-like tumors, and TNBC was used as a surrogate to represent the basal-like category of breast cancer, which may confound the results of our investigation to some extent. Secondly, the immunostaining data did not classify TNBC into detailed subtypes in the present study. Thirdly, we acknowledge other limitations, including the retrospective study design, small sample size used and short follow-up periods. Finally, other unbalanced confounding factors between groups, including hormone therapy regimen, economic conditions, smoking, alcohol consumption, obesity and chronic disease, were also potential limitations. As a result, the contribution of the present study to the investigation of molecular subtypes in breast cancer may be limited.

In conclusion, the data in the present study suggests that the clinicopathological characteristics of TNBC differ from luminal and HER2-enriched subtypes. TNBC patients treated with BCT did not have a significantly increased risk of recurrence or mortality, and patients with TNBC may remain appropriate candidates for BCT. However, detailed molecular taxonomy and prospective randomized clinical trials are required.

\section{Acknowledgements}

The present study was partly supported by the Grant from National Natural Science Foundation of China (grant no. 81472472) and Grant from the Clinical New Technology Development Project from the Tianjin Medical University Cancer Institute and Hospital (grant no. 2012017).

\section{References}

1. Wiechmann L, Sampson M, Stempel M, Jacks LM, Patil SM, King T and Morrow M: Presenting features of breast cancer differ by molecular subtype. Ann Surg Oncol 16: 2705-2710, 2009.

2. Perou CM: Molecular stratification of triple-negative breast cancers. Oncologist 1 (16 Suppl): S61-S70, 2011

3. Perou CM, Sorlie T, Eisen MB, van de Rijn M, Jeffrey SS, Rees CA, Pollack JR, Ross DT, Johnsen H, Akslen LA, et al: Molecular portraits of human breast tumours. Nature 406: 747-752, 2000

4. Abramson VG, Lehmann BD, Ballinger TJ and Pietenpol JA: Subtyping of triple-negative breast cancer: Implications for therapy. Cancer 121: 8-16, 2015.

5. Foulkes WD, Smith IE and Reis-Filho JS: Triple-negative breast cancer. N Engl JMed 363: 1938-1948, 2010.

6. Brenton JD, Carey LA, Ahmed AA and Caldas C: Molecular classification and molecular forecasting of breast cancer: Ready for clinical application? J Clin Oncol 23: 7350-7360, 2005 .

7. Pignol JP, Rakovitch E and Olivotto IA: Is breast conservation therapy superior to mastectomy for women with triple-negative breast cancers? J Clin Oncol 29: 2841-2843, 2011.

8. Dent R, Trudeau M, Pritchard KI, Hanna WM, Kahn HK, Sawka CA, Lickley LA, Rawlinson E, Sun P and Narod SA: Triple-negative breast cancer: Clinical features and patterns of recurrence. Clin Cancer Res 13: 4429-4434, 2007.

9. Kyndi M, Sørensen FB, Knudsen H, Overgaard M, Nielsen HM and Overgaard J; Danish Breast Cancer Cooperative Group: Estrogen receptor, progesterone receptor, HER-2, and response to postmastectomy radiotherapy in high-risk breast cancer: The danish breast cancer cooperative group. J Clin Oncol 26: 1419-1426, 2008.

10. Voduc KD, Cheang MC, Tyldesley S, Gelmon K, Nielsen TO and Kennecke H: Breast cancer subtypes and the risk of local and regional relapse. J Clin Oncol 28: 1684-1691, 2010.
11. Gabos Z, Thoms J, Ghosh S, Hanson J, Deschenes J, Sabri S and Abdulkarim B: The association between biological subtype and locoregional recurrence in newly diagnosed breast cancer. Breast Cancer Res Treat 124: 187-194, 2010.

12. van Dongen JA, Voogd AC, Fentiman IS, Legrand C, Sylvester RJ, Tong D, van der Schueren E, Helle PA, van Zijl K and Bartelink H: Long-term results of a randomized trial comparing breast-conserving therapy with mastectomy: European organization for research and treatment of cancer 10801 trial. J Natl Cancer Inst 92: 1143-1150, 2000.

13. Veronesi U, Cascinelli N, Mariani L, Greco M, Saccozzi R, Luini A, Aguilar M and Marubini E: Twenty-year follow-up of a randomized study comparing breast-conserving surgery with radical mastectomy for early breast cancer. N Engl J Med 347: 1227-1232, 2002.

14. Lazovich D, Solomon CC, Thomas DB, Moe RE and White E: Breast conservation therapy in the United States following the 1990 national institutes of health consensus development conference on the treatment of patients with early stage invasive breast carcinoma. Cancer 86: 628-637, 1999.

15. Nguyen PL, Taghian AG, Katz MS, Niemierko A, Abi Raad RF, Boon WL, Bellon JR, Wong JS, Smith BL and Harris JR: Breast cancer subtype approximated by estrogen receptor, progesterone receptor and HER-2 is associated with local and distant recurrence after breast-conserving therapy. J Clin Oncol 26: 2373-2378, 2008

16. Solin LJ, Hwang WT and Vapiwala N: Outcome after breast conservation treatment with radiation for women with triple-negative early-stage invasive breast carcinoma. Clin Breast Cancer 9: 96-100, 2009.

17. Haffty BG, Yang Q, Reiss M, Kearney T, Higgins SA, Weidhaas J, Harris L, Hait W and Toppmeyer D: Locoregional relapse and distant metastasis in conservatively managed triple negative early-stage breast cancer. J Clin Oncol 24: 5652-5657, 2006.

18. Millar EK, Graham PH, O'Toole SA, McNeil CM, Browne L, Morey AL, Eggleton S, Beretov J, Theocharous C, Capp A, et al: Prediction of local recurrence, distant metastases and death after breast-conserving therapy in early-stage invasive breast cancer using a five-biomarker panel. J Clin Oncol 27: 4701-4708, 2009.

19. Gangi A, Chung A, Mirocha J, Liou DZ, Leong T and Giuliano AE: Breast-conserving therapy for triple-negative breast cancer. JAMA Surg 149: 252-258, 2014.

20. Connolly JL: Changes and problematic areas in interpretation of the AJCC cancer staging manual, 6th edition, for breast cancer. Arch Pathol Lab Med 130: 287-291, 2006.

21. Elston CW and Ellis IO: Pathological prognostic factors in breast cancer. I. The value of histological grade in breast cancer: Experience from a large study with long-term follow-up. Histopathology 19: 403-410, 1991.

22. Tummaruk P, Tienthai P, Manee-In S and Srisuwatanasagul S: Expression of progesterone receptor in the utero-tubal junction after intra-uterine and deep intra-uterine insemination in sows. Reprod Domest Anim 45: e26-e31, 2010.

23. Cejalvo JM, Martínez de Dueñas E, Galván P, García-Recio S, Burgués Gasión O, Paré L, Antolín S, Martinello R, Blancas I, Adamo B, et al: Intrinsic subtypes and gene expression profiles in primary and metastatic breast cancer. Cancer Res 77: 2213-2221, 2017.

24. Kaplan HG, Malmgren JA and Atwood M: T1N0 triple negative breast cancer: Risk of recurrence and adjuvant chemotherapy. Breast J 15: 454-460, 2009.

25. Noh JM, Choi DH, Huh SJ, Park W, Yang JH, Nam SJ, Im YH and Ahn JS: Patterns of recurrence after breast-conserving treatment for early stage breast cancer by molecular subtype. J Breast Cancer 14: 46-51, 2011.

26. Kim JY, Chang SK, Park H, Lee BM and Shin HS: Treatment outcome in patients with triple negative early stage breast cancers compared with other molecular subtypes. Radiat Oncol J 30: 124-131, 2012.

27. Freedman GM, Anderson PR, Li T and Nicolaou N: Locoregional recurrence of triple-negative breast cancer after breast-conserving surgery and radiation. Cancer 115: 946-951, 2009.

28. Braunstein LZ, Niemierko A, Shenouda MN, Truong L, Sadek BT, Abi Raad R, Wong JS, Punglia RS, Taghian AG and Bellon JR: Outcome following local-regional recurrence in women with early-stage breast cancer: Impact of biologic subtype. Breast J 21: 161-167, 2015. 
29. Abdulkarim BS, Cuartero J, Hanson J, Deschenes J, Lesniak D and Sabri S: Increased risk of locoregional recurrence for women with T1-2N0 triple-negative breast cancer treated with modified radical mastectomy without adjuvant radiation therapy compared with breast-conserving therapy. J Clin Oncol 29: 2852-2858, 2011.

30. Wang J, Shi M, Ling R, Xia Y, Luo S, Fu X, Xiao F, Li J, Long X, Wang J, et al: Adjuvant chemotherapy and radiotherapy in triple-negative breast carcinoma: A prospective randomized controlled multi-center trial. Radiother Oncol 100: 200-204, 2011.

31. Guiu S, Michiels S, Andre F, Cortes J, Denkert C, Di Leo A, Hennessy BT, Sorlie T, Sotiriou C, Turner N, et al: Molecular subclasses of breast cancer: How do we define them? The IMPAKT 2012 working group statement. Ann Oncol 23: 2997-3006, 2012.

32. Weigelt B, Mackay A, A'Hern R, Natrajan R, Tan DS, Dowsett M, Ashworth A and Reis-Filho JS: Breast cancer molecular profiling with single sample predictors: A retrospective analysis. Lancet Oncol 11: 339-349, 2010.
33. Lehmann BD, Bauer JA, Chen X, Sanders ME, Chakravarthy AB, Shyr Y and Pietenpol JA: Identification of human triple-negative breast cancer subtypes and preclinical models for selection of targeted therapies. J Clin Invest 121: 2750-2767, 2011.

34. Eiermann W and Vallis KA: Locoregional treatments for triple-negative breast cancer. Ann Oncol 23 (Suppl 6): vi30-vi34, 2012.

35. Sotiriou C, Neo SY, McShane LM, Korn EL, Long PM, Jazaeri A, Martiat P, Fox SB, Harris AL and Liu ET: Breast cancer classification and prognosis based on gene expression profiles from a population-based study. Proc Natl Acad Sci USA 100: 10393-10398, 2003. 